\title{
On floors and ceilings of the $k$-Catalan arrangement
}

\author{
Marko Thiel \\ Fakultät für Mathematik \\ Universität Wien \\ Wien, Austria \\ marko.thiel@univie.ac.at
}

Submitted: Feb 28, 2014; Accepted: Nov 6, 2014; Published: Nov 13, 2014

Mathematics Subject Classifications: 17B22; 20F55; 52C35

\begin{abstract}
The set of dominant regions of the $k$-Catalan arrangement of a crystallographic root system $\Phi$ is a well-studied object enumerated by the Fuß-Catalan number $C a t^{(k)}(\Phi)$. It is natural to refine this enumeration by considering floors and ceilings of dominant regions. A conjecture of Armstrong states that counting dominant regions by their number of floors of a certain height gives the same distribution as counting dominant regions by their number of ceilings of the same height. We prove this conjecture using a bijection that provides even more refined enumerative information.
\end{abstract}

Keywords: Fuss-Catalan combinatorics; Catalan arrangement; Floors; Ceilings

\section{Introduction}

Let $\Phi$ be a crystallographic root system of rank $n$ with simple system $S$, positive system $\Phi^{+}$, and ambient vector space $V$. For background on root systems see [Hum90]. For $k$ a positive integer, we define the $k$-Catalan arrangement of $\Phi$ as the hyperplane arrangement given by the hyperplanes $H_{\alpha}^{r}=\{x \in V \mid\langle x, \alpha\rangle=r\}$ for $\alpha \in \Phi$ and $r \in\{0,1, \ldots, k\}$. The complement of this arrangement falls apart into connected components which we call the regions of the arrangement. Those regions $R$ that have $\langle x, \alpha\rangle>0$ for all $\alpha \in \Phi^{+}$and all $x \in R$ we call dominant. The number of dominant regions of the $k$-Catalan arrangement equals the Fuß-Catalan number $\mathrm{Cat}^{(k)}(\Phi)$ [Ath04] of $\Phi$. This number remains somewhat mysterious, in the sense that it also counts other objects in combinatorics, like the set of $k$-divisible noncrossing partitions $N C^{(k)}(\Phi)$ of $\Phi$ [Arm09, Theorem 3.5.3] and the number of facets of the $k$-generalised cluster complex $\Delta^{(k)}(\Phi)$ of $\Phi$ [FR05, Proposition 8.4], but no uniform proof of this fact is known, that is every known proof of this fact appeals to 
the classification of irreducible crystallographic root systems.

For a dominant region $R$ of the $k$-Catalan arrangement, we call those hyperplanes that support a facet of $R$ the walls of $R$. Those walls of $R$ which do not contain the origin and have the origin on the same side as $R$ we call the ceilings of $R$. The walls of $R$ that do not contain the origin and separate $R$ from the origin are called its floors. We say a hyperplane is of height $r$ if it is of the form $H_{\alpha}^{r}$ for $\alpha \in \Phi^{+}$.

One reason why floors and ceilings of dominant regions are interesting is that they give a more refined enumeration of the dominant regions of the $k$-Catalan arrangement of $\Phi$ that corresponds to refined enumerations of other objects counted by the Fuß-Catalan number $\operatorname{Cat}^{(k)}(\Phi)$. More precisely, the number of dominant regions in the $k$-Catalan arrangement of $\Phi$ that have exactly $j$ floors of height $k$ equals the Fuß-Narayana number $\operatorname{Nar}^{(k)}(\Phi, j)$ [Ath05, Proposition 5.1] [Thi14, Theorem 1], which also counts the number of $k$-divisible noncrossing partitions of $\Phi$ of rank $j$ [Arm09, Definition 3.5.4], as well as equalling the $(n-j)$-th entry of the $h$-vector of the $k$-generalised cluster complex $\Delta^{(k)}(\Phi)$ [FR05, Theorem 10.2]. Similarly, the number of bounded dominant regions of the $k$ Catalan arrangement of $\Phi$ that have exactly $j$ ceilings of height $k$ equals the $(n-j)$-th entry of the $h$-vector of the positive part of $\Delta^{(k)}(\Phi)$ [AT06, Conjecture 1.2] [Thi14, Corollary 5].

For the special case where $\Phi$ is of type $A_{n-1}$, more is known. For example, there is an explicit bijection between the set of dominant regions of the $k$-Catalan arrangement of $\Phi$ and the set of facets of the cluster complex of $\Phi$ [FKT13]. There is also an enumeration of those dominant regions that have a fixed hyperplane as a floor [FTV13]. In contrast to those results, all results in this paper are stated and proven uniformly for all crystallographic root systems without appeal to the classification.

If $M$ is any set of hyperplanes of the $k$-Catalan arrangement, let $U(M)$ be the set of dominant regions $R$ of the $k$-Catalan arrangement such that all hyperplanes in $M$ are floors of $R$. Similarly, let $L(M)$ be the set of dominant regions $R^{\prime}$ of the $k$-Catalan arrangement such that all hyperplanes in $M$ are ceilings of $R^{\prime}$. Use the standard notation $[n]:=\{1,2, \ldots, n\}$. Then we have the following theorem.

Theorem 1.1. For any set $M=\left\{H_{\alpha_{1}}^{i_{1}}, H_{\alpha_{2}}^{i_{2}}, \ldots, H_{\alpha_{m}}^{i_{m}}\right\}$ of $m$ hyperplanes with $i_{j} \in[k]$ and $\alpha_{j} \in \Phi^{+}$for all $j \in[m]$, there is an explicit bijection $\Theta$ from $U(M)$ to $L(M)$.

See Figure 1 for an example. From this theorem, we obtain some enumerative corollaries. In particular, let $f l_{r}(l)$ be the number of dominant regions in the $k$-Catalan arrangement that have exactly $l$ floors of height $r$, and let $c l_{r}(l)$ be the number of dominant regions that have exactly $l$ ceilings of height $r$ [Arm09, Definition 5.1.23]. We deduce the following conjecture of Armstrong.

Corollary 1.2 ([Arm09, Conjecture 5.1.24]). We have $f l_{r}(l)=c l_{r}(l)$ for all $1 \leqslant r \leqslant k$ and $0 \leqslant l \leqslant n$. 

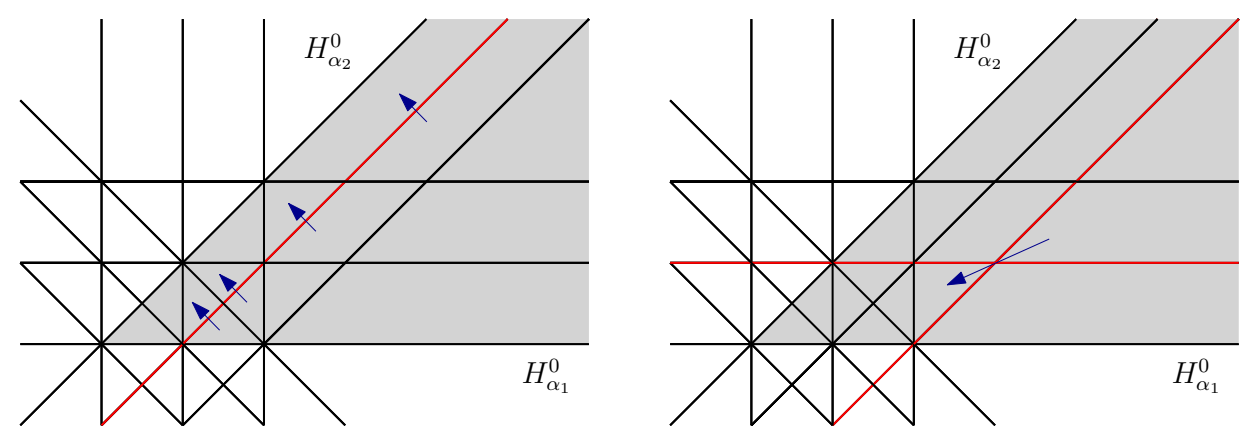

Figure 1: The bijection $\Theta$ for the 2-Catalan arrangement of the root system of type $B_{2}$, for $M=\left\{H_{\alpha_{2}}^{1}\right\}$ and for $M=\left\{H_{\alpha_{1}}^{1}, H_{\alpha_{2}}^{2}\right\}$. The dominant chamber is shaded in grey.

Specialising to the $k=1$ case, we also give a geometric interpretation in terms of dominant regions of the Catalan arrangement of the Panyushev complement on ideals in the root poset of $\Phi$.

\section{Definitions}

For this section and the next one, suppose that $\Phi$ is irreducible. Define the affine Coxeter arrangement of $\Phi$ as the union of all hyperplanes of the form $H_{\alpha}^{r}=\{x \in V \mid\langle x, \alpha\rangle=r\}$ for $\alpha \in \Phi$ and $r \in \mathbb{Z}$. Then the complement of this falls apart into connected components, all of which are congruent open $n$-simplices, called alcoves. The affine Weyl group $W_{a}$ generated by all the reflections through hyperplanes of the form $H_{\alpha}^{r}$ for $\alpha \in \Phi$ and $r \in \mathbb{Z}$ is a Coxeter group, with generating set $S_{a}=\left\{s_{0}, s_{1}, \ldots, s_{n}\right\}$, where $s_{1}, \ldots, s_{n}$ are the reflections in the hyperplanes orthogonal to the simple roots of $\Phi$ and $s_{0}$ is the reflection in $H_{\tilde{\alpha}}^{1}$, where $\tilde{\alpha}$ is the highest root of $\Phi$.

The group $W_{a}$ acts simply transitively on the alcoves, so if we define the fundamental alcove as

$$
A_{\circ}=\left\{x \in V \mid\left\langle x, \alpha_{i}\right\rangle>0 \text { for all } \alpha_{i} \in S,\langle x, \tilde{\alpha}\rangle<1\right\},
$$

then every alcove $A$ can be written as $w\left(A_{\circ}\right)$ for a unique $w \in W_{a}$.

Clearly any alcove is contained in exactly one region $R$ of the $k$-Catalan arrangement of $\Phi$. For any alcove $A$ in the affine Coxeter arrangement of $\Phi$ and $\alpha \in \Phi^{+}$, there exists a unique integer $r$ with $r-1<\langle x, \alpha\rangle<r$ for all $x \in A$. We denote this integer by $r(A, \alpha)$.

Suppose that for each $\alpha \in \Phi^{+}$we are given a positive integer $r_{\alpha}$. The following is due to Shi [Shi87, Theorem 5.2].

Lemma 2.1 ([AT06, Lemma 2.3]). There is an alcove $A$ with $r(A, \alpha)=r_{\alpha}$ for all $\alpha \in \Phi^{+}$ if and only if $r_{\alpha}+r_{\beta}-1 \leqslant r_{\alpha+\beta} \leqslant r_{\alpha}+r_{\beta}$ whenever $\alpha, \beta, \alpha+\beta \in \Phi^{+}$. 
Define a partial order on $\Phi^{+}$by

$$
\alpha \leqslant \beta \text { if and only if } \beta-\alpha \in\langle S\rangle_{\mathbb{N}},
$$

that is, $\beta \geqslant \alpha$ if and only if $\beta-\alpha$ can be written as a linear combination of simple roots with nonnegative integer coefficients. The set of positive roots $\Phi^{+}$with this partial order is called the root poset. A subset $I \subseteq \Phi^{+}$is called an ideal if for all $\alpha \in I$ and $\beta \leqslant \alpha$, also $\beta \in I$. A subset $J \subseteq \Phi^{+}$is called an order filter if for all $\alpha \in J$ and $\beta \geqslant \alpha$, also $\beta \in J$.

Suppose $\mathcal{I}=\left(I_{1}, I_{2}, \ldots, I_{k}\right)$ is an ascending (multi)chain of $k$ ideals in the root poset of $\Phi$, that is $I_{1} \subseteq I_{2} \subseteq \ldots \subseteq I_{k}$. Setting $J_{i}=\Phi^{+} \backslash I_{i}$ for $i \in[k]$ and $\mathcal{J}=\left(J_{1}, J_{2}, \ldots, J_{k}\right)$ gives us the corresponding descending chain of order filters. That is, we have $J_{1} \supseteq J_{2} \supseteq \ldots \supseteq J_{k}$. The ascending chain of ideals $\mathcal{I}$ and the corresponding descending chain of order filters $\mathcal{J}$ are both called geometric if the following conditions are satisfied simultaneously.

1. $\left(I_{i}+I_{j}\right) \cap \Phi^{+} \subseteq I_{i+j}$ for all $i, j \in\{0,1, \ldots, k\}$ with $i+j \leqslant k$, and

2. $\left(J_{i}+J_{j}\right) \cap \Phi^{+} \subseteq J_{i+j}$ for all $i, j \in\{0,1, \ldots, k\}$.

Here we set $I_{0}=\varnothing, J_{0}=\Phi^{+}$, and $J_{i}=J_{k}$ for $i>k$. We call $\mathcal{I}$ and $\mathcal{J}$ positive if $S \subseteq I_{k}$, or equivalently $S \cap J_{k}=\varnothing$.

Let $R$ be a dominant region of the $k$-Catalan arrangement of $\Phi$. Let us define $\theta(R)=$ $\left(I_{1}, I_{2}, \ldots, I_{k}\right)$ and $\phi(R)=\left(J_{1}, J_{2}, \ldots, J_{k}\right)$, where

$$
\begin{gathered}
I_{i}=\left\{\alpha \in \Phi^{+} \mid\langle x, \alpha\rangle<i \text { for all } x \in R\right\} \text { and } \\
J_{i}=\left\{\alpha \in \Phi^{+} \mid\langle x, \alpha\rangle>i \text { for all } x \in R\right\},
\end{gathered}
$$

for $i \in\{0,1, \ldots, k\}$. It is not difficult to verify that $\theta(R)$ is a geometric chain of ideals and that $\phi(R)$ is the corresponding geometric chain of order filters.

For a geometric chain of ideals $\mathcal{I}=\left(I_{1}, I_{2}, \ldots, I_{k}\right)$, and $\alpha \in \Phi^{+}$, we define

$$
r_{\alpha}(\mathcal{I})=\min \left\{r_{1}+r_{2}+\ldots+r_{m} \mid \alpha=\alpha_{1}+\alpha_{2}+\ldots+\alpha_{m} \text { and } \alpha_{i} \in I_{r_{i}} \text { for all } i \in[m]\right\}
$$

where we set $r_{\alpha}(\mathcal{I})=\infty$ if $\alpha$ cannot be written as a linear combination of elements in $I_{k}$. So $r_{\alpha}(\mathcal{I})<\infty$ for all $\alpha \in \Phi^{+}$if and only if $\mathcal{I}$ is positive.

For a geometric chain of order filters $\mathcal{J}=\left(J_{1}, J_{2}, \ldots, J_{k}\right)$, and $\alpha \in \Phi^{+}$, we define

$$
k_{\alpha}(\mathcal{J})=\max \left\{k_{1}+k_{2}+\ldots+k_{m} \mid \alpha=\alpha_{1}+\alpha_{2}+\ldots+\alpha_{m} \text { and } \alpha_{i} \in J_{k_{i}} \text { for all } i \in[m]\right\} \text {, }
$$

where $k_{i} \in\{0,1, \ldots, k\}$ for all $i \in[m]$.

It turns out that $\phi$ is a bijection from the set of dominant regions of the $k$-Catalan arrangement of $\Phi$ to the set of geometric chains of $k$ order filters in the root poset of 
$\Phi[$ Ath05, Theorem 3.6]. Its inverse $\psi$ is the map sending a geometric chain of order filters $\mathcal{J}$ to the region $R$ of the $k$-Catalan arrangement containing the alcove $A$ with $r(A, \alpha)=k_{\alpha}(\mathcal{J})+1$ for all $\alpha \in \Phi^{+}$. This alcove $A$ is called the minimal alcove of $R$. Its floors are exactly the floors of $R$ [Ath05, Theorem 3.11].

Thus the map $\theta$ is a bijection from dominant regions $R$ of the $k$-Catalan arrangement to geometric chains of ideals $\mathcal{I}$. It restricts to a bijection between bounded dominant regions of the $k$-Catalan arrangement and positive geometric chains of ideals. The inverse of this restriction maps a positive geometric chain of ideals $\mathcal{I}$ to the bounded dominant region $R$ in the $k$-Catalan arrangement containing the alcove $B$ with $r(B, \alpha)=r_{\alpha}(\mathcal{I})$ for all $\alpha \in \Phi^{+}$[AT06, Theorem 3.6]. This alcove $B$ is called the maximal alcove of $R$. Its ceilings are exactly the ceilings of $R$ [AT06, Theorem 3.11].

We call $\alpha \in \Phi^{+}$a rank $r$ indecomposable element [Ath05, Definition 3.8] of a geometric chain of order filters $\mathcal{J}=\left(J_{1}, J_{2}, \ldots, J_{k}\right)$ if $\alpha \in J_{r}$ and

1. $k_{\alpha}(\mathcal{J})=r$,

2. $\alpha \notin J_{i}+J_{j}$ for $i+j=r$ and

3. if $k_{\alpha+\beta}(\mathcal{J})=t \leqslant k$ for some $\beta \in \Phi^{+}$then $\beta \in J_{t-r}$.

We have that $H_{\alpha}^{r}$ is a floor of $R$ if and only if $\alpha$ is a rank $r$ indecomposable element of the geometric chain of order filters $\mathcal{J}=\phi(R)$ [Ath05, Theorem 3.11].

We call $\alpha \in \Phi^{+}$a rank $r$ indecomposable element [AT06, Definition 3.8] of a geometric chain of ideals $\mathcal{I}=\left(I_{1}, I_{2}, \ldots, I_{k}\right)$ if $\alpha \in I_{r}$ and

1. $r_{\alpha}(\mathcal{I})=r$,

2. $\alpha \notin I_{i}+I_{j}$ for $i+j=r$ and

3. if $r_{\alpha+\beta}(\mathcal{I})=t \leqslant k$ for some $\beta \in \Phi^{+}$then $\beta \in I_{t-r}$.

We will soon see that $H_{\alpha}^{r}$ is a ceiling of $R$ if and only if $\alpha$ is a rank $r$ indecomposable element of the geometric chain of ideals $\mathcal{I}=\theta(R)$.

\section{Lemmas}

Our aim for this rather technical section is to prove the following theorem.

Theorem 3.1. Let $R$ be a dominant region in the $k$-Catalan arrangement of $\Phi, \mathcal{I}=\theta(R)$ and $\alpha \in \Phi^{+}$. Then $R$ contains an alcove $B$ such that for all $r \in[k]$ the following are equivalent: 
1. $H_{\alpha}^{r}$ is a ceiling of $R$,

2. $\alpha$ is a rank $r$ indecomposable element of $\mathcal{I}$, and

3. $H_{\alpha}^{r}$ is a ceiling of $B$.

It is already known that Theorem 3.1 holds for bounded dominant regions [AT06, Theorem 3.11]. In that case, we may take the alcove $B$ to be the maximal alcove of the bounded region $R$.

Our approach to proving Theorem 3.1 is to note that when a region $R$ of the $k$-Catalan arrangement is subdivided into regions of the $(k+1)$-Catalan arrangement by hyperplanes of the form $H_{\alpha}^{k+1}$ for $\alpha \in \Phi^{+}$, at least one of the resulting regions is bounded. We find a region $\underline{R}$ of the $(k+1)$-Catalan arrangement which, among the bounded regions of the $(k+1)$-Catalan arrangement that are contained in $R$, is the one furthest away from the origin. We call the maximal alcove $B$ of $\underline{R}$ the pseudomaximal alcove of $R$. It equals the maximal alcove of $R$ if $R$ is bounded. The alcove $B \subseteq R$ will be seen to satisfy the assertion of Theorem 3.1. Instead of working directly with the dominant regions of the $k$ - and $(k+1)$-Catalan arrangements, we usually phrase our results in terms of the corresponding geometric chains of ideals.

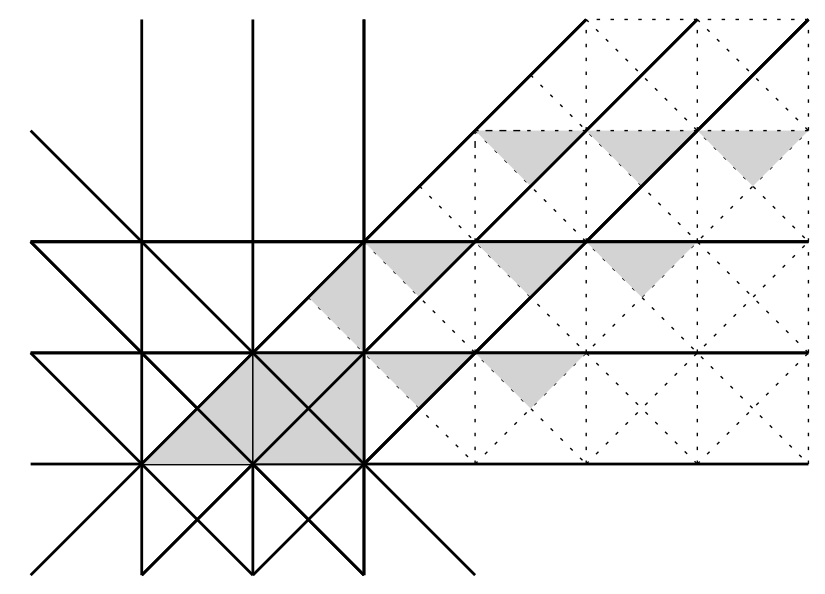

Figure 2: The dominant regions of the 2-Catalan arrangement of the root system of type $B_{2}$ together with their pseudomaximal alcove, shaded in grey.

We require the following lemmas:

Lemma 3.2 ([Ath05, Lemma 2.1 (ii)]). If $\alpha_{1}, \alpha_{2}, \ldots, \alpha_{r} \in \Phi$ and $\alpha_{1}+\alpha_{2}+\ldots+\alpha_{r}=$ $\alpha \in \Phi$, then $\alpha_{1}=\alpha$ or there exists $i$ with $2 \leqslant i \leqslant r$ such that $\alpha_{1}+\alpha_{i} \in \Phi \cup\{0\}$.

Lemma 3.3 ([AT06, Lemma 3.2]). For $\alpha \in \Phi^{+}$and $r_{\alpha}(\mathcal{I})=r \leqslant k$, we have that $\alpha \in I_{r}$.

Lemma 3.4 ([AT06, Lemma 3.10]). Suppose $\alpha$ is an indecomposable element of $\mathcal{I}$. Then 


$$
\begin{aligned}
& \text { 1. } r_{\alpha}(\mathcal{I})=r_{\beta}(\mathcal{I})+r_{\gamma}(\mathcal{I})-1 \text { if } \alpha=\beta+\gamma \text { for } \beta, \gamma \in \Phi^{+} \text {and } \\
& \text { 2. } r_{\alpha}(\mathcal{I})+r_{\beta}(\mathcal{I})=r_{\alpha+\beta}(\mathcal{I}) \text { if } \beta, \alpha+\beta \in \Phi^{+} \text {. }
\end{aligned}
$$

Lemma 3.5. If $\alpha, \beta, \gamma \in \Phi^{+}, \beta+\gamma \in \Phi^{+}$and $\alpha \leqslant \beta+\gamma$, then $\alpha \leqslant \beta$ or $\alpha \leqslant \gamma$ or $\alpha=\beta^{\prime}+\gamma^{\prime}$ with $\beta^{\prime}, \gamma^{\prime} \in \Phi^{+}, \beta^{\prime} \leqslant \beta$ and $\gamma^{\prime} \leqslant \gamma$.

Proof. Let $\alpha=\beta+\gamma-\sum_{j \in J} \alpha_{j}$ with $\alpha_{j} \in S$ for all $j \in J$. We proceed by induction on $|J|$. If $|J|=0$, we are done. If $|J|=1$, we have that $\alpha=-\alpha_{i}+\beta+\gamma$ for some $\alpha_{i} \in S$. Thus by Lemma 3.2, we have either $\alpha=-\alpha_{i}$ (a contradiction), or $\beta^{\prime}=\beta-\alpha_{i} \in \Phi \cup\{0\}$ or $\gamma^{\prime}=\gamma-\alpha_{i} \in \Phi \cup\{0\}$. Notice that if $\beta^{\prime} \neq 0$, then $\beta^{\prime} \in \Phi^{+}$, and similarly for $\gamma^{\prime}$. So if $\beta^{\prime} \in \Phi^{+}$we may write $\alpha=\beta^{\prime}+\gamma$ and otherwise we have $\gamma^{\prime} \in \Phi^{+}$and thus $\alpha=\beta+\gamma^{\prime}$ as required.

If $|J|>1$, we have $\alpha+\sum_{j \in J} \alpha_{j}=\beta+\gamma$, so by Lemma 3.2, either $\alpha=\beta+\gamma$, so we are done, or $\alpha+\alpha_{j} \in \Phi \cup\{0\}$ for some $j \in J$. In the latter case we even have $\alpha+\alpha_{j} \in \Phi^{+}$. By induction hypothesis, $\alpha+\alpha_{j} \leqslant \beta$ or $\alpha+\alpha_{j} \leqslant \gamma$ or $\alpha+\alpha_{j}=\beta^{\prime}+\gamma^{\prime}$ with $\beta^{\prime}, \gamma^{\prime} \in \Phi^{+}, \beta^{\prime} \leqslant \beta$ and $\gamma^{\prime} \leqslant \gamma$. In the first two cases, we are done. In the latter case, we have $\alpha=-\alpha_{j}+\beta^{\prime}+\gamma^{\prime}$, so we proceed as in the $|J|=1$ case.

We are now ready to define the bounded dominant region $\underline{R}$ of the $(k+1)$-Catalan arrangement in terms of the corresponding geometric chain of $k+1$ ideals $\mathcal{I}$. For a geometric chain of ideals $\mathcal{I}=\left(I_{1}, I_{2}, \ldots, I_{k}\right)$, let $\underline{I}_{i}=I_{i}$ for all $i \in[k]$ and let $\underline{I}_{k+1}=$ $\bigcup_{i+j=k+1}\left(\left(I_{i}+I_{j}\right) \cap \Phi^{+}\right) \cup I_{k} \cup S$. By Lemma $3.5, \underline{I}_{k+1}$ is an ideal. Define $\underline{\mathcal{I}}=\left(\underline{I}_{1}, \ldots, \underline{I}_{k+1}\right)$.

Lemma 3.6. If $\mathcal{I}=\left(I_{1}, I_{2}, \ldots, I_{k}\right)$ is a geometric chain of $k$ ideals in the root poset of $\Phi$, then $\underline{I}$ is a positive geometric chain of $k+1$ ideals. The bounded dominant region $\underline{R}=\theta^{-1}(\underline{\mathcal{I}})$ of the $(k+1)$-Catalan arrangement of $\Phi$ is contained in the region $R=\theta^{-1}(\mathcal{I})$ of the $k$-Catalan arrangement.

Proof. By construction, $\mathcal{I}$ is an ascending chain of ideals. If $i+j \leqslant k$, we have that $\left(\underline{I}_{i}+\underline{I}_{j}\right) \cap \Phi^{+}=\left(I_{i}+I_{j}\right) \cap \Phi^{+} \subseteq I_{i+j}=\underline{I}_{i+j}$ as $\mathcal{I}$ is geometric. If $i+j=k+1$ with $i, j \neq 0$ (otherwise the result is trivial) we have that $\left(\underline{I}_{i}+\underline{I}_{j}\right) \cap \Phi^{+}=\left(I_{i}+I_{j}\right) \cap \Phi^{+} \subseteq$ $\bigcup_{i+j=k+1}\left(\left(I_{i}+I_{j}\right) \cap \Phi^{+}\right) \subseteq \underline{I}_{i+j}$.

Let $\mathcal{J}=\left(J_{1}, J_{2}, \ldots, J_{k}\right)$ be the geometric chain of order filters corresponding to the geometric chain of ideals $\mathcal{I}$. Define $\underline{\mathcal{J}}$ similarly. We need to verify that $\left(\underline{J}_{i}+\underline{J}_{j}\right) \cap \Phi^{+} \subseteq \underline{J}_{i+j}$ for all $i, j \in[k+1]$.

Suppose first that $i+j \leqslant k$. Then $\left(\underline{J}_{i}+\underline{J}_{j}\right) \cap \Phi^{+}=\left(J_{i}+J_{j}\right) \cap \Phi^{+} \subseteq J_{i+j}=\underline{J}_{i+j}$ since $\mathcal{J}$ is geometric.

Suppose next that $i+j=k+1$. Take any region $R^{\prime}$ of the $(k+1)$-Catalan arrangement that is contained in $R$. Let $\theta\left(R^{\prime}\right)=\mathcal{I}^{\prime}=\left(I_{1}^{\prime}, I_{2}^{\prime}, \ldots, I_{k+1}^{\prime}\right)$ be the geometric chain 
of ideals corresponding to $R^{\prime}$ and let $\mathcal{J}^{\prime}=\left(J_{1}^{\prime}, J_{2}^{\prime}, \ldots, J_{k+1}^{\prime}\right)$ be the corresponding geometric chain of order filters. Then $R$ and $R^{\prime}$ are on the same side of each hyperplane of the $k$-Catalan arrangement. Thus $I_{l}^{\prime}=I_{l}$ and $J_{l}^{\prime}=J_{l}$ for $l \in[k]$. Thus we have $\underline{I}_{k+1}=$ $\bigcup_{i+j=k+1}\left(\left(I_{i}+I_{j}\right) \cap \Phi^{+}\right) \cup I_{k} \cup S=\bigcup_{i+j=k+1}\left(\left(I_{i}^{\prime}+I_{j}^{\prime}\right) \cap \Phi^{+}\right) \cup I_{k}^{\prime} \cup S \subseteq I_{k+1}^{\prime} \cup S$ since $\mathcal{I}^{\prime}$ is geometric. Since $\mathcal{J}^{\prime}$ is geometric, we have $\left(\underline{J}_{i}+\underline{J}_{j}\right) \cap \Phi^{+}=\left(J_{i}^{\prime}+J_{j}^{\prime}\right) \cap \Phi^{+} \subseteq J_{i+j}^{\prime}=J_{k+1}^{\prime}$. The sum of two positive roots is never a simple root, so we even have $\left(\underline{J}_{i}+\underline{J}_{j}\right) \cap \Phi^{+} \subseteq J_{k+1}^{\prime} \backslash S$. But $J_{k+1}^{\prime} \backslash S \subseteq \underline{J}_{k+1}$, as $\underline{I}_{k+1} \subseteq I_{k+1}^{\prime} \cup S$. Thus $\left(\underline{J}_{i}+\underline{J}_{j}\right) \cap \Phi^{+} \subseteq \underline{J}_{i+j}$.

Lastly, in the case where $i+j>k+1$, we have $\underline{J}_{j} \subseteq \underline{J}_{k+1-i}$, so that $\left(\underline{J}_{i}+\underline{J}_{j}\right) \cap \Phi^{+} \subseteq$ $\left(\underline{J}_{i}+\underline{J}_{k+1-i}\right) \cap \Phi^{+} \subseteq \underline{J}_{k+1}=\underline{J}_{i+j}$.

Thus the chain of ideals $\underline{\mathcal{I}}$ is geometric. It is also clearly positive, so $\underline{R}=\theta^{-1}(\underline{\mathcal{I}})$ is bounded. Since $\underline{I}_{i}=I_{i}$ for $i \in[k], \underline{R}$ and $R$ are on the same side of each hyperplane of the $k$-Catalan arrangement, so $\underline{R}$ is contained in $R$.

For a geometric chain of $k$ ideals $\mathcal{I}=\left(I_{1}, I_{2}, \ldots, I_{k}\right)$, define $\operatorname{supp}(\mathcal{I})=I_{k} \cap S$. In particular, $\operatorname{supp}(\mathcal{I})=S$ if and only if $\mathcal{I}$ is positive.

Lemma 3.7. If $\alpha \in\langle\operatorname{supp}(\mathcal{I})\rangle_{\mathbb{N}}$, then $r_{\alpha}(\underline{\mathcal{I}})=r_{\alpha}(\mathcal{I})$. In particular, if $r_{\alpha}(\underline{\mathcal{I}}) \leqslant k$, then $r_{\alpha}(\underline{\mathcal{I}})=r_{\alpha}(\mathcal{I})$.

Proof. First note that $\alpha \in\langle\operatorname{supp}(\mathcal{I})\rangle_{\mathbb{N}}$ implies that $r_{\alpha}(\mathcal{I})<\infty$. So may write $\alpha=$ $\alpha_{1}+\alpha_{2}+\ldots+\alpha_{m}$ with $\alpha_{i} \in I_{r_{i}}$ for $i \in[m]$ and $r_{1}+r_{2}+\ldots+r_{m}=r_{\alpha}(\mathcal{I})$. Since $\alpha_{i} \in I_{r_{i}}=\underline{I}_{r_{i}}$ this implies that $r_{\alpha}(\underline{\mathcal{I}}) \leqslant r_{\alpha}(\mathcal{I})$.

We may write $\alpha=\alpha_{1}+\alpha_{2}+\ldots+\alpha_{m}$ with $\alpha_{i} \in \underline{I}_{r_{i}}$ for $i \in[m]$ and $r_{1}+r_{2}+\ldots+r_{m}=r_{\alpha}(\underline{\mathcal{I}})$. We wish to show that $r_{\alpha}(\mathcal{I}) \leqslant r_{\alpha}(\underline{I})$. Thus we seek to write $\alpha=\alpha_{1}^{\prime}+\alpha_{2}^{\prime}+\ldots+\alpha_{l}^{\prime}$ with $\alpha_{i}^{\prime} \in I_{r_{i}^{\prime}}$ for $i \in[l]$ and $r_{1}^{\prime}+r_{2}^{\prime}+\ldots+r_{l}^{\prime}=r_{\alpha}(\underline{\mathcal{I}})$. If $r_{p}=k+1$ for some $p \in[m]$, then $\alpha_{p} \in \underline{I}_{k+1}=\bigcup_{i+j=k+1}\left(\left(I_{i}+I_{j}\right) \cap \Phi^{+}\right) \cup I_{k} \cup S$. If $\alpha_{p} \in I_{k}=\underline{I}_{k}$, we get a contradiction with the minimality of $r_{\alpha}(\underline{I})$. If $\alpha_{p} \in S$, then since $\alpha_{p} \in\langle\operatorname{supp}(\mathcal{I})\rangle_{\mathbb{N}}$, we have that $\alpha_{p} \in \operatorname{supp}(\mathcal{I}) \subseteq I_{k}$, again a contradiction. So $\alpha_{p} \in \bigcup_{i+j=k+1}\left(\left(I_{i}+I_{j}\right) \cap \Phi^{+}\right)$. Thus write $\alpha_{p}=\beta_{p}+\beta_{p}^{\prime}$, where $\beta_{p} \in I_{i}$ and $\beta_{p}^{\prime} \in I_{j}$ for some $i, j$ with $i+j=k+1$. So in the sum $\alpha=\alpha_{1}+\alpha_{2}+\ldots+\alpha_{m}$ replace each $\alpha_{p}$ with $r_{p}=k+1$ with $\beta_{p}+\beta_{p}^{\prime}$ to obtain (after renaming) $\alpha=\alpha_{1}^{\prime}+\alpha_{2}^{\prime}+\ldots+\alpha_{l}^{\prime}$ with $\alpha_{i}^{\prime} \in I_{r_{i}^{\prime}}$ for $i \in[l]$ and $r_{1}^{\prime}+r_{2}^{\prime}+\ldots+r_{l}^{\prime}=r_{\alpha}(\underline{\mathcal{I}})$, as required.

If $r_{\alpha}(\underline{\mathcal{I}})=r \leqslant k$, then $\alpha \in I_{r} \subseteq I_{k}$ by Lemma 3.3, so $\alpha \in\langle\operatorname{supp}(\mathcal{I})\rangle_{\mathbb{N}}$ and thus $r_{\alpha}(\underline{\mathcal{I}})=r_{\alpha}(\mathcal{I})$.

For $R$ a dominant region of the $k$-Catalan arrangement, define the pseudomaximal alcove of $R$ to be the maximal alcove of $\underline{R}$. This term is justified by the following proposition.

Proposition 3.8. If $R$ is a bounded dominant region of the $k$-Catalan arrangement, its pseudomaximal alcove is equal to its maximal alcove.

Proof. Let $A$ and $B$ be the maximal and pseudomaximal alcoves of $R$ respectively. If $\mathcal{I}=\theta(R)$, then $r(\alpha, A)=r_{\alpha}(\mathcal{I})$ for all $\alpha \in \Phi^{+}$. Since $B$ is the maximal alcove of $\underline{R}$, 
we have $r(\alpha, B)=r_{\alpha}(\underline{\mathcal{I}})$ for all $\alpha \in \Phi^{+}$. Now $\mathcal{I}$ is positive since $R$ is bounded, so $\operatorname{supp}(\mathcal{I})=S$. Thus $r_{\alpha}(\mathcal{I})=r_{\alpha}(\underline{\mathcal{I}})$ for all $\alpha \in \Phi^{+}$by Lemma 3.7. So $r(\alpha, A)=r(\alpha, B)$ for all $\alpha \in \Phi^{+}$and therefore $A=B$.

Lemma 3.9. Let $R$ be a region of the $k$-Catalan arrangement of $\Phi$, let be $B$ be its pseudomaximal alcove and let $t \leqslant k$ be a positive integer. If $\left\langle x_{0}, \alpha\right\rangle>t$ for some $x_{0} \in R$, then $\langle x, \alpha\rangle>t$ for all $x \in B$.

Proof. Let $\mathcal{I}=\theta(R)$. Since $r(B, \alpha)=r_{\alpha}(\underline{\mathcal{I}})$ for all $\alpha \in \Phi^{+}$, it suffices to show that $r_{\alpha}(\underline{\mathcal{I}})>t$. If $r_{\alpha}(\underline{\mathcal{I}})>k$ this is immediate, so we may assume that $r_{\alpha}(\underline{\mathcal{I}}) \leqslant k$. Thus we have $r_{\alpha}(\underline{\mathcal{I}})=r_{\alpha}(\mathcal{I})$ by Lemma 3.7. Write $\alpha=\alpha_{1}+\alpha_{2}+\ldots+\alpha_{m}$, with $\alpha_{i} \in I_{r_{i}}$ for all $i \in[m]$ and $r_{1}+r_{2}+\ldots+r_{m}=r_{\alpha}(\mathcal{I})$. Then $\left\langle x, \alpha_{i}\right\rangle<r_{i}$ for all $i \in[m]$ and $x \in R$, so $\langle x, \alpha\rangle<r_{\alpha}(\mathcal{I})$ for all $x \in R$. So if $\left\langle x_{0}, \alpha\right\rangle>t$ for some $x_{0} \in R$, then $r_{\alpha}(\mathcal{I})>\left\langle x_{0}, \alpha\right\rangle>t$, so $r_{\alpha}(\underline{\mathcal{I}})=r_{\alpha}(\mathcal{I})>t$.

Lemma 3.10. If $\alpha$ is a rank $r$ indecomposable element of $\mathcal{I}$, then $\alpha$ is a rank $r$ indecomposable element of $\underline{\mathcal{I}}$.

Proof. Let $\alpha$ be a rank $r$ indecomposable element of $\mathcal{I}$. Then $\alpha \in I_{r}=\underline{I}_{r}$, and $r_{\alpha}(\underline{\mathcal{I}})=$ $r_{\alpha}(\mathcal{I})=r$ by Lemma 3.7. We have that $\alpha \notin I_{i}+I_{j}=\underline{I}_{i}+\underline{I}_{j}$ for $i+j=r$. If $r_{\alpha+\beta}(\underline{\mathcal{I}})=t \leqslant k+1$, then $\alpha+\beta \in \underline{I}_{t}$ by Lemma 3.3. So if $t \leqslant k$, we have $r_{\alpha+\beta}(\mathcal{I})=r_{\alpha+\beta}(\underline{\mathcal{I}})$ by Lemma 3.7. If $t=k+1$, then $\alpha+\beta \in I_{k}$ or $\alpha+\beta \in \bigcup_{i+j=k+1}\left(\left(I_{i}+I_{j}\right) \cap \Phi^{+}\right)$, since $\alpha+\beta \notin S$. Either way, $\alpha+\beta \in\left\langle I_{k}\right\rangle_{\mathbb{N}}$ so $r_{\alpha+\beta}(\mathcal{I})=r_{\alpha+\beta}(\underline{\mathcal{I}})$ by Lemma 3.7. Thus we have $r_{\alpha}(\mathcal{I})+r_{\beta}(\mathcal{I})=r_{\alpha+\beta}(\mathcal{I})=r_{\alpha+\beta}(\underline{\mathcal{I}})=t$ using Lemma 3.4. So $r_{\beta}(\mathcal{I})=t-r_{\alpha}(\mathcal{I})=t-r$, so $\beta \in I_{t-r}=\underline{I}_{t-r}$ by Lemma 3.3. Thus $\alpha$ is a rank $r$ indecomposable element of $\underline{\mathcal{I}}$.

Lemma 3.11. If $\alpha \in \Phi^{+}$and $H_{\alpha}^{r}$ is a ceiling of a dominant region $R$ of the $k$-Catalan arrangement, then $\alpha$ is a rank $r$ indecomposable element of $\mathcal{I}=\theta(R)$.

Proof. Since the origin and $R$ are on the same side of $H_{\alpha}^{r}$, we have that $\langle x, \alpha\rangle<r$ for all $x \in R$, so $\alpha \in I_{r}$ and thus $r_{\alpha}(\mathcal{I}) \leqslant r$. But if $r_{\alpha}(\mathcal{I})=i<r$, then $\alpha \in I_{i}$ by Lemma 3.3, so $\langle x, \alpha\rangle<i \leqslant r-1$ for all $x \in R$. So $H_{\alpha}^{r}$ is not a wall of $R$, a contradiction. Thus $r_{\alpha}(\mathcal{I})=r$.

If $\alpha=\beta+\gamma$ for $\beta \in I_{i}$ and $\gamma \in I_{j}$ with $i+j=r$, then the fact that $\langle x, \alpha\rangle<r$ for all $x \in R$ is a consequence of $\langle x, \beta\rangle<i$ and $\langle x, \gamma\rangle<j$ for all $x \in R$, so $H_{\alpha}^{r}$ does not support a facet of $R$. So $\alpha \notin I_{i}+I_{j}$ for $i+j=r$.

If $r_{\alpha+\beta}(\mathcal{I})=t \leqslant k$, then $\alpha+\beta \in I_{t}$ by Lemma 3.3, so $\langle x, \alpha+\beta\rangle<t$ for all $x$ in $R$. If also $\langle x, \beta\rangle>t-r$ for all $x \in R$, then $\langle x, \alpha\rangle\langle r$ for all $x \in R$ is a consequence of these, so $H_{\alpha}^{r}$ does not support a facet of $R$. So $\langle x, \beta\rangle<t-r$ for all $x \in R$, so $\beta \in I_{t-r}$.

Thus $\alpha$ is a rank $r$ indecomposable element of $\mathcal{I}$. 
Proof of Theorem 3.1. We take $B$ to be the pseudomaximal alcove of $R$, that is the maximal alcove of $\underline{R}$. We will show that $(1) \Rightarrow(2) \Rightarrow(3) \Rightarrow(1)$.

The statement that $(1) \Rightarrow(2)$ is Lemma 3.11 .

For $(2) \Rightarrow(3)$, suppose $\alpha$ is a rank $r$ indecomposable element of $\mathcal{I}$. Then by Lemma 3.10, $\alpha$ is also a rank $r$ indecomposable element of $\underline{\mathcal{I}}$. So by Lemma 3.4, we have $r_{\alpha}(\underline{\mathcal{I}})=r_{\beta}(\underline{\mathcal{I}})+r_{\gamma}(\underline{\mathcal{I}})-1$ if $\alpha=\beta+\gamma$ for $\beta, \gamma \in \Phi^{+}$, and also $r_{\alpha}(\underline{\mathcal{I}})+r_{\beta}(\underline{\mathcal{I}})=r_{\alpha+\beta}(\underline{\mathcal{I}})$ if $\beta, \alpha+\beta \in \Phi^{+}$. Thus there exists an alcove $B^{\prime}$ with $r\left(B^{\prime}, \beta\right)=r_{\beta}(\underline{\mathcal{I}})$ for $\beta \neq \alpha$ and $r\left(B^{\prime}, \alpha\right)=r_{\alpha}(\underline{\mathcal{I}})+1$ by Lemma 2.1. Since $r(B, \beta)=r_{\beta}(\underline{\mathcal{I}})$ for all $\beta \in \Phi^{+}$, this means that $B^{\prime}$ and $B$ are on the same side of each hyperplane of the affine Coxeter arrangement, except for $H_{\alpha}^{r_{\alpha}(\mathcal{I})}=H_{\alpha}^{r}$. Thus $H_{\alpha}^{r}$ is a wall of $B$. Since $H_{\alpha}^{r}$ does not separate $B$ from the origin, it is a ceiling of $B$.

For $(3) \Rightarrow(1)$, suppose $H_{\alpha}^{r}$ is a ceiling of $B$. Let $B^{\prime}$ be the alcove which is the reflection of $B$ in the hyperplane $H_{\alpha}^{r}$. Then $\langle x, \alpha\rangle>r$ for all $x \in B^{\prime}$, so by Lemma 3.9 the alcove $B^{\prime}$ is not contained in $R$. Thus $H_{\alpha}^{r}$ is a wall of $R$. It does not separate $R$ from the origin, so it is a ceiling of $R$. This completes the proof.

\section{Proof of Theorem 1.1}

We are now in a position to prove Theorem 1.1.

Proof of Theorem 1.1. Let us at first suppose that $\Phi$ is an irreducible crystallographic root system of rank $n$. For $m=0$, the statement is immediate. Suppose that $0<m \leqslant n$.

To define the bijection $\Theta$, let $R \in U(M)$ and let $A$ be the minimal alcove of $R$. The reflections $s_{\alpha_{1}}^{i_{1}}, \ldots, s_{\alpha_{m}}^{i_{m}}$ in the hyperplanes $H_{\alpha_{1}}^{i_{1}}, \ldots, H_{\alpha_{m}}^{i_{m}}$ are reflections in facets of the alcove $A=w\left(A_{\circ}\right)$, so the set $S^{\prime}=\left\{s_{\alpha_{1}}^{i_{1}}, \ldots, s_{\alpha_{m}}^{i_{m}}\right\}$ equals $w J w^{-1}$ for some $J \subset S_{a}$ and $w \in W_{a}$. Thus the reflection group $W^{\prime}$ generated by $S^{\prime}$ is a proper parabolic subgroup of $W_{a}$. In particular, it is finite. With respect to the finite reflection group $W^{\prime}$, the alcove $A$ is contained in the dominant Weyl chamber, that is the set

$$
C=\left\{x \in V \mid\left\langle x, \alpha_{j}\right\rangle>i_{j} \text { for all } j \in[m]\right\} .
$$

So if $w_{0}^{\prime}$ is the longest element of $W^{\prime}$ with respect to the generating set $S^{\prime}$, the alcove $A^{\prime}=w_{0}^{\prime}(A)$ is contained in the Weyl chamber

$$
w_{0}^{\prime}(C)=\left\{x \in V \mid\left\langle x, \alpha_{j}\right\rangle<i_{j} \text { for all } j \in[m]\right\}
$$

of $W^{\prime}$, so it is on the other side of all the hyperplanes $H_{\alpha_{1}}^{i_{1}}, \ldots, H_{\alpha_{m}}^{i_{m}}$. $A^{\prime}$ is an alcove, so it is contained in some region $R^{\prime}$. Set $\Theta(R)=R^{\prime}$.

Claim 1. The region $R^{\prime}$ is dominant and all hyperplanes in $M$ are ceilings of $R^{\prime}$, that is $R^{\prime} \in L(M)$, so $\Theta$ is well-defined. 


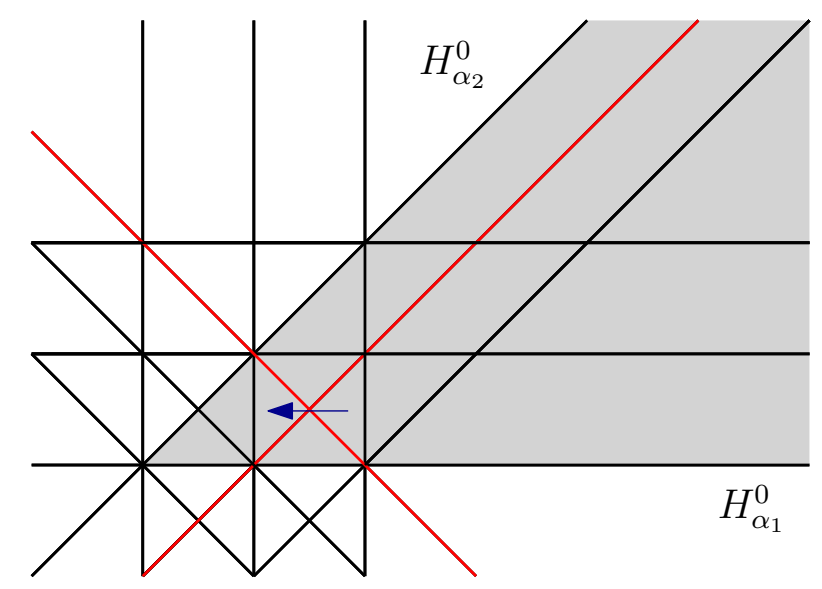

Figure 3: The bijection $\Theta$ for the 2-Catalan arrangement of the root system of type $B_{2}$ with $M=\left\{H_{\alpha_{2}}^{1}, H_{2 \alpha_{1}+\alpha_{2}}^{2}\right\}$.

Proof. The origin is contained in the Weyl chamber $w_{0}^{\prime}(C)$ of $W^{\prime}$. Thus no reflection in $W^{\prime}$ fixes the origin. We can write $A^{\prime}=w_{0}^{\prime}(A)$ as $t_{r} \cdots t_{1}(A)$ where $t_{i} \in W^{\prime}$ is a reflection in a facet of $t_{i-1} \cdots t_{1}(A)$ for all $i \in[r]$. In fact, if $w_{0}^{\prime}=s_{1}^{\prime} \cdots s_{r}^{\prime}$ with $s_{i}^{\prime} \in S^{\prime}$ for all $i \in[r]$ is a reduced expression for $w_{0}^{\prime}$ in $W^{\prime}$, we can take $t_{i}=s_{1}^{\prime} \cdots s_{i-1}^{\prime} s_{i}^{\prime} s_{i-1}^{\prime} \cdots s_{1}^{\prime}$. So $t_{i} \cdots t_{1}(A)$ and $t_{i-1} \cdots t_{1}(A)$ are on the same side of every hyperplane in the affine Coxeter arrangement of $\Phi$ except for the reflecting hyperplane of $t_{i}$. Since $t_{i}$ does not fix the origin, if $t_{i-1} \cdots t_{1}(A)$ is dominant, then so is $t_{i} \cdots t_{1}(A)$. Thus by induction on $i$, the alcove $A^{\prime}$ is dominant, so $R^{\prime}$ is dominant.

Consider the Coxeter arrangement of $W^{\prime}$, which is the hyperplane arrangement given by the reflecting hyperplanes of all the reflections in $W^{\prime}$. The action of $W^{\prime}$ on $V$ restricts to an action on the set of these hyperplanes. Since $H_{\alpha_{1}}^{i_{1}}, \ldots, H_{\alpha_{m}}^{i_{m}}$ support facets of $A$, $w_{0}^{\prime}\left(H_{\alpha_{1}}^{i_{1}}\right), \ldots, w_{0}^{\prime}\left(H_{\alpha_{m}}^{i_{m}}\right)$ support facets of $A^{\prime}=w_{0}^{\prime}(A)$. Now the set $\left\{w_{0}^{\prime}\left(H_{\alpha_{1}}^{i_{1}}\right), \ldots, w_{0}^{\prime}\left(H_{\alpha_{m}}^{i_{m}}\right)\right\}$ is the set of walls of $w_{0}^{\prime}(C)$ in the Coxeter arrangement of $W^{\prime}$, so it equals the set $M=\left\{H_{\alpha_{1}}^{i_{1}}, \ldots, H_{\alpha_{m}}^{i_{m}}\right\}$. Since all hyperplanes in $M$ are floors of $A$, and $A^{\prime}$ is on the other side of each of them, they are all ceilings of $A^{\prime}$. Thus they are ceilings of $R^{\prime}$.

We show that $\Theta$ is a bijection by exhibiting its inverse $\Psi$, a map from $L(M)$ to $U(M)$. Suppose $R^{\prime} \in L(M)$. Let $B$ be the alcove in $R^{\prime}$ given by Theorem 3.1. Let $R^{\prime \prime}$ be the region that contains $B^{\prime}=w_{0}^{\prime}(B)$. Similarly to the proof of Claim 1, we have that $R^{\prime \prime} \in U(M)$. So let $\Psi\left(R^{\prime}\right)=R^{\prime \prime}$.

Claim 2. The maps $\Theta$ and $\Psi$ are inverse to each other, so $\Theta$ is a bijection.

Proof. Suppose $R \in U(M), R^{\prime}=\Theta(R)$ and $R^{\prime \prime}=\Psi\left(R^{\prime}\right)$. Use the same notation as above for the alcoves $A, A^{\prime}, B$ and $B^{\prime}$. Suppose for contradiction that $R^{\prime \prime} \neq R$. Then there is a hyperplane $H=H_{\alpha}^{r}$ of the $k$-Catalan arrangement that separates $R$ and $R^{\prime \prime}$. So $H$ separates $A$ and $B^{\prime}$. Now $A$ and $B^{\prime}$ are in the dominant Weyl chamber of $W^{\prime}$, so they 
are on the same side of each reflecting hyperplane of $W^{\prime}$. Thus $H$ is not a reflecting hyperplane of $W^{\prime}$. Now we may write $A^{\prime}$ as $t_{r} \cdots t_{1}(A)$, where $t_{i} \in W^{\prime}$ is a reflection in a facet of $t_{i-1} \cdots t_{1}(A)$ for all $i \in[r]$. So $t_{i} \cdots t_{1}(A)$ and $t_{i-1} \cdots t_{1}(A)$ are on the same side of every hyperplane in the affine Coxeter arrangement, except for the reflecting hyperplane of $t_{i}$, which cannot be $H$. Thus by induction on $i$, the alcove $A^{\prime}$ is on the same side of $H$ as $A$. Similarly $B$ is on the same side of $H$ as $B^{\prime}$. So $A^{\prime}$ and $B$ are on different sides of $H$, a contradiction, as they are contained in the same region, namely $R^{\prime}$. Thus $\Psi(\Theta(R))=R^{\prime \prime}=R$, so $\Psi \circ \Theta=i d$. Similarly $\Theta \circ \Psi=i d$, so $\Theta$ and $\Psi$ are inverse to each other, so $\Theta$ is a bijection.

For any dominant alcove, at least one of its $n+1$ facets must either be a floor or contain the origin, and at least one must be a ceiling. So it has at most $n$ ceilings and at most $n$ floors. So any dominant region $R$ of the $k$-Catalan arrangement has at most $n$ ceilings and at most $n$ floors. Thus if $m>n$, both $U(M)$ and $L(M)$ are empty. This completes the proof in the case where $\Phi$ is irreducible.

Now suppose $\Phi$ is reducible, say $\Phi=\Phi_{1} \amalg \Phi_{2}$ with $\Phi_{1} \perp \Phi_{2}$. So $V=V_{1} \oplus V_{2}$ with $V_{1}=\left\langle\Phi_{1}\right\rangle$ and $V_{2}=\left\langle\Phi_{2}\right\rangle$, and $V_{1} \perp V_{2}$. Then the regions of the $k$-Catalan arrangement of $\Phi$ are precisely the sets of the form $R_{1} \oplus R_{2}$ where $R_{i}$ is a region of the $k$-Catalan arrangement of $\Phi_{i}$ for $i=1,2$. The region $R_{1} \oplus R_{2}$ is dominant if and only if $R_{1}$ and $R_{2}$ are both dominant. A hyperplane $H_{\alpha}^{r}$ is a floor of $R_{1} \oplus R_{2}$ if and only if $H_{\alpha}^{r}$ is a floor of $R_{i}$ for some $i=1,2$. The same holds for ceilings. Say $M=M_{1} \amalg M_{2}$ with $H_{\alpha_{j}}^{i_{j}} \in M_{i}$ if $\alpha_{j} \in \Phi_{i}$ for $j \in[m]$ and $i=1,2$. Assume the theorem holds for $\Phi_{1}$ and $\Phi_{2}$, giving us bijections $\Theta_{1}$ and $\Theta_{2}$ for $\Phi_{1}$ together with $M_{1}$ and $\Phi_{2}$ together with $M_{2}$ respectively. Then $\Theta\left(R_{1} \oplus R_{2}\right)=\Theta_{1}\left(R_{1}\right) \oplus \Theta_{2}\left(R_{2}\right)$ gives the required bijection for $\Phi$ together with $M$. This completes the proof by induction on the number of irreducible components of $\Phi$.

\section{Corollaries}

We deduce some enumerative corollaries of Theorem 1.1. For any set $M$ of hyperplanes of the $k$-Catalan arrangement, let $U_{=}(M)$ be the set of dominant regions $R$ of the $k$-Catalan arrangement such that the floors of $R$ are exactly the hyperplanes in $M$, and let $L_{=}(M)$ be the set of dominant regions $R^{\prime}$ of the $k$-Catalan arrangement such that the ceilings of $R^{\prime}$ are exactly the hyperplanes in $M$.

Corollary 5.1. For any set $M=\left\{H_{\alpha_{1}}^{i_{1}}, H_{\alpha_{2}}^{i_{2}}, \ldots, H_{\alpha_{m}}^{i_{m}}\right\}$ of $m$ hyperplanes with $i_{j} \in[k]$ and $\alpha_{j} \in \Phi^{+}$for all $j \in[m]$, we have that $\left|U_{=}(M)\right|=\left|L_{=}(M)\right|$.

Proof. This follows from Theorem 1.1 by an application of the Principle of Inclusion and Exclusion.

Corollary 5.2. For any tuple $\left(a_{1}, a_{2}, \ldots, a_{k}\right)$ of nonnegative integers, the number of dominant regions $R$ that have exactly $a_{j}$ floors of height $j$ for all $j \in[k]$ is the same as the number of dominant regions $R^{\prime}$ that have exactly $a_{j}$ ceilings of height $j$ for all $j \in[k]$. 
Proof. Sum Corollary 5.1 over all sets $M$ containing exactly $a_{j}$ hyperplanes of height $j$ for all $j \in[k]$.

Proof of Corollary 1.2. Set $a_{r}=l$ and sum Corollary 5.2 over all choices of $a_{j}$ for all $j \neq r$.

\section{The Panyushev complement}

In the special case where $k=1$, a geometric chain of ideals $\mathcal{I}$ is simply the single ideal $I_{1}$, similarly a geometric chain of order filters $\mathcal{J}$ is just the single order filter $J_{1}$. The indecomposable elements of an ideal $I$ are then just its maximal elements [AT06, Lemma 3.9]. The indecomposable elements of an order filter $J$ are just its minimal elements [Ath05, Lemma 3.9] [Thi14, Lemma 1].

There is a natural bijection between ideals and antichains of any poset that sends an ideal to the set of its maximal elements. Similarly, there is a natural bijection between order filters and antichains that sends an order filter to the set of its minimal elements.

So for an ideal $I$ in the root poset of $\Phi$, we define the Panyushev complement $\operatorname{Pan}(I)$ as the ideal generated by the minimal elements of the order filter $J=\Phi^{+} \backslash I$. From the above considerations, this is a bijection from the set of order ideals of the root poset of $\Phi$ to itself.

For a region $R$ of the Catalan arrangement, let

$$
\begin{gathered}
C L(R)=\left\{\alpha \in \Phi^{+} \mid H_{\alpha}^{1} \text { is a ceiling of } R\right\} \text { and } \\
F L(R)=\left\{\alpha \in \Phi^{+} \mid H_{\alpha}^{1} \text { is a floor of } R\right\} .
\end{gathered}
$$

Since a region $R$ in the Catalan arrangement corresponds to a unique ideal $I=\theta(R)$, which corresponds uniquely to the set of its maximal elements, which equals $C L(R)$ by Theorem 3.1, the map $C L: R \mapsto C L(R)$ gives a bijection from the set of dominant regions in the Catalan arrangement to the set of antichains in the root poset. That the same holds for the map $F L: R \mapsto F L(R)$ follows from an analogous argument that can already be deduced from [Ath05, Theorem 3.11].

Theorem 6.1. For an ideal $I$ in the root poset of $\Phi$, the region $\theta^{-1}(\operatorname{Pan}(I))$ is the unique region of the Catalan arrangement of $\Phi$ whose ceilings are exactly the floors of the region $\theta^{-1}(I)$.

Proof. The set $C L\left(\theta^{-1}(\operatorname{Pan}(I))\right)$ is the set of maximal elements of $\operatorname{Pan}(I)$, which equals the set of minimal elements of $J=\Phi^{+} \backslash I$, which equals $F L\left(\theta^{-1}(I)\right)$. Since $C L$ is a bijection, $\theta^{-1}(\operatorname{Pan}(I))$ is the only region $R^{\prime}$ with $C L\left(R^{\prime}\right)=F L\left(\theta^{-1}(I)\right)$.

We could rephrase Theorem 6.1 as $\operatorname{Pan}=\theta \circ C L^{-1} \circ F L \circ \theta^{-1}$. The fact that the Panyushev complement has a natural interpretation in terms of the dominant regions of the Catalan arrangement may serve to explain why it seems to be of particular interest for root posets. 


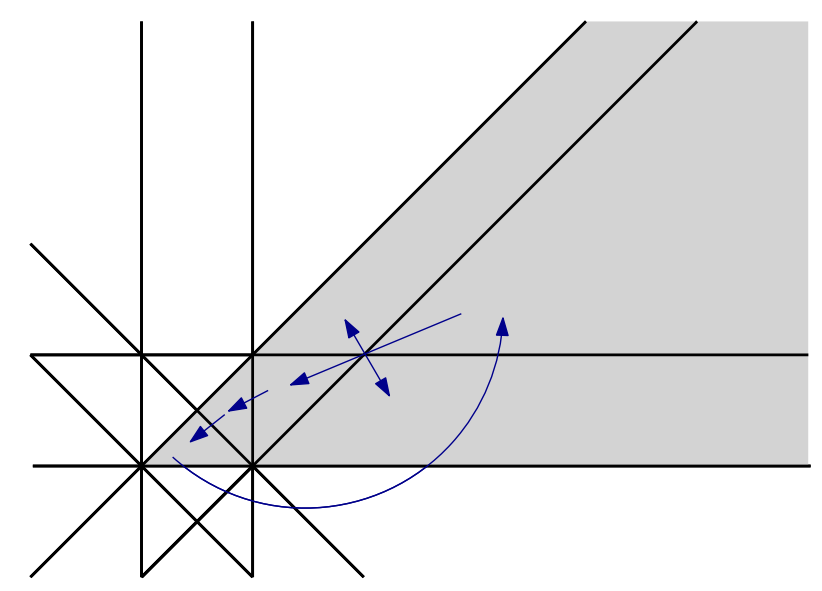

Figure 4: The action of $\theta^{-1} \circ \operatorname{Pan} \circ \theta=C L^{-1} \circ F L$ on the dominant regions of the Catalan arrangement of the root system of type $B_{2}$.

\section{Acknowledgements}

I thank Myrto Kallipoliti, Henri Mühle, Vivien Ripoll and Eleni Tzanaki for helpful comments and suggestions. I also wish to express my gratitude to the anonymous referee for a very careful reading of the manuscript.

\section{References}

[Arm09] Drew Armstrong. Generalized Noncrossing Partitions and Combinatorics of Coxeter Groups. Memoirs of the American Mathematical Society, 202, 2009.

[AT06] Christos A. Athanasiadis and Eleni Tzanaki. On the enumeration of positive cells in generalized cluster complexes and Catalan hyperplane arrangements. Journal of Algebraic Combinatorics, 23:355-375, 2006.

[Ath04] Christos A. Athanasiadis. Generalized Catalan Numbers, Weyl Groups and Arrangements of Hyperplanes. Bulletin of the London Mathematical Society, 36:294-302, 2004.

[Ath05] Christos A. Athanasiadis. On a Refinement of the Generalized Catalan Numbers for Weyl Groups. Transactions of the American Mathematical Society, 357:179196, 2005.

[FKT13] Susanna Fishel, Myrto Kallipoliti, and Eleni Tzanaki. Facets of the generalized cluster complex and regions in the extended Catalan arrangement of type $A_{n}$. The Electronic Journal of Combinatorics, 20(4):\#P7, 2013.

[FR05] Sergey Fomin and Nathan Reading. Generalized Cluster Complexes and Coxeter Combinatorics. International Mathematics Research Notices, 44:2709-2757, 2005. 
[FTV13] Susanna Fishel, Eleni Tzanaki, and Monica Vazirani. Counting Shi regions with a fixed separating wall. Annals of Combinatorics, 17:671-693, 2013.

[Hum90] James E. Humphreys. Reflection Groups and Coxeter Groups. Cambridge University Press, Cambridge, 1990.

[Shi87] Jian-yi Shi. Alcoves corresponding to an affine Weyl group. Journal of the London Mathematical Society, 35:42-55, 1987.

[Thi14] Marko Thiel. On the $H$-triangle of generalised nonnesting partitions. European Journal of Combinatorics, 39:244-255, 2014. 